\title{
Toward a Core Domain Set for Glucocorticoid Impact in Inflammatory Rheumatic Diseases: The OMERACT 2018 Glucocorticoid Impact Working Group
}

\author{
Jonathan T.L. Cheah (D), Rachel J. Black (i), Joanna C. Robson (1), Iris Y. Navarro-Millán (D), \\ Sarah R. Young, Pamela Richards, Susan Beard, Lee S. Simon, Susan M. Goodman (D), \\ Sarah L. Mackie (D), and Catherine L. Hill (D)
}

ABSTRACT. Objective. To understand the effects of glucocorticoids (GC), which are of importance to patients. Methods. The results of 2 literature reviews, a patient survey, and a qualitative study were presented. Results. No validated instrument exists to evaluate GC effect on patients. Survey data revealed skin thinning/bruising, sleep disturbance, and weight gain as the most frequent adverse effects. The qualitative research yielded rich data covering rapid benefits and physical and emotional consequences of GC.

Conclusion. It was agreed that a patient-reported outcome to measure GC effect was required and a research agenda was developed for this goal. (First Release January 15 2019; J Rheumatol 2019; 46:1179-82; doi:10.3899/jrheum.181082)

Key Indexing Terms:

OMERACT

GLUCOCORTICOIDS

OUTCOME ASSESSMENT

RHEUMATIC DISEASES

ADVERSE EFFECTS

From the Division of Rheumatology, Hospital for Special Surgery;

Department of Medicine, and the Division of General Internal Medicine,

Weill Cornell Medicine, New York; Department of Social Work,

Binghamton University, Binghamton, New York; SDG LLC, Cambridge,

Massachusetts, USA; Rheumatology Unit, Royal Adelaide Hospital;

Discipline of Medicine, The University of Adelaide; Rheumatology Unit,

The Queen Elizabeth Hospital, Adelaide, Australia; Faculty of Health and Applied Science, University of the West of England; School of Clinical

Science, University of Bristol, Bristol; Leeds Biomedical Research Centre,

Leeds Institute of Rheumatic and Musculoskeletal Medicine; Chapel

Allerton Hospital, University of Leeds, Leeds, UK.

INM was supported by grant K23AR068449 from the US National Institute of Arthritis and Musculoskeletal and Skin Diseases.

J.T. Cheah, MBBS, Fellow, Division of Rheumatology, Hospital for Special Surgery, and Department of Medicine, Weill Cornell Medicine; R.J. Black, MBBS, Consultant Rheumatologist, Rheumatology Unit, Royal Adelaide Hospital, and Discipline of Medicine, The University of Adelaide; J.C. Robson, MBBS, PhD, MRCP, Consultant Senior Lecturer in Rheumatology, Faculty of Health and Applied Science, University of the West of England, and School of Clinical Science, University of Bristol; I.Y. Navarro-Millán, MD, MSPH, Assistant Professor of Medicine. Division of General Internal Medicine, Weill Cornell Medicine, and Division of Rheumatology, Hospital for Special Surgery; S.R. Young, PhD, LMSW, Assistant Professor, Department of Social Work, Binghamton University; P. Richards, HNC (Business Studies), OMERACT Patient Research Partner; S. Beard, BMus, OMERACT Patient Research Partner; L.S. Simon, MD, Principal, SDG LLC; S.M. Goodman, MD, Professor of Clinical Medicine, Division of Rheumatology, Hospital for Special Surgery, and Department of Medicine, Weill Cornell Medicine;

S.L. Mackie, MB BCh, PhD, MRCP, Leeds Biomedical Research Centre,

Leeds Institute of Rheumatic and Musculoskeletal Medicine, Chapel Allerton Hospital, and University of Leeds; C.L. Hill, MBBS, MSc, MD,

FRACP, Clinical Professor, Rheumatology Unit, The Queen Elizabeth

Hospital, and Rheumatology Unit, Royal Adelaide Hospital, and

Discipline of Medicine, The University of Adelaide.

Address correspondence to Dr. J.T. Cheah, Division of Rheumatology,

Hospital for Special Surgery, 535 E. 70th St., New York, New York 10021,

USA.E-mail: cheahj@hss.edu

Accepted for publication November 27, 2018.
Glucocorticoids (GC) have a substantial role in the treatment of inflammatory diseases ${ }^{1,2}$. However, while adverse effects (AE) are well documented, the absolute risk of many GC AE remains unquantified ${ }^{3}$ and the effects of greatest importance to patients are not known. With the aim to define a research agenda for measuring the effect of GC to identify relevant domains, the Outcome Measures in Rheumatology (OMERACT) GC Impact Working Group (WG) held its inaugural meeting at the 2016 OMERACT meeting ${ }^{4}$. The presented work included a literature search that confirmed that there was not an already developed patient-reported outcome (PRO) for the effects of systemic GC use. Additionally, the preliminary results of a pilot survey and 2 qualitative studies identified that patients are concerned and affected by outcomes that are not commonly assessed by their treating clinician, such as skin fragility, sleep disturbance, and weight gain. At that meeting, there was agreement on the need for a data-driven PRO identifying both positive and negative effects of GC therapy to be used across inflammatory conditions. However, it was agreed that further work was required to gain additional understanding of the effects of GC prior to the development of a PRO.

Following the steps of the current OMERACT filter ${ }^{5,6}$, we sought to generate candidate domains from which to propose a core domain set. To this end, 2 literature reviews, a cross-sectional survey, and a qualitative study were undertaken and presented at OMERACT 2018 to better understand the effect of GC across various patient groups.

Personal non-commercial use only. The Journal of Rheumatology Copyright $\subset$ (2019. All rights reserved. 


\section{MATERIALS AND METHODS/RESULTS}

Systematic literature review (SLR) of PRO for the effect of systemic GC. At OMERACT 2016, a systematic review revealed that no PRO had been developed to assess the effect of systemic GC use. We updated the original search, using OVID MEDLINE (2013 to Week 1 of October 2017) and OVID EMBASE (1974 to October 16, 2017). There were 208 unique articles identified and screened. Although no PRO measuring the effects of systemic GC across all inflammatory diseases were identified, 2 disease-specific PRO were found: 1 for multiple sclerosis (MS) and 1 for systemic lupus erythematosus (SLE) ${ }^{7,8}$. The Methylprednisolone Adverse Effects Questionnaire assessed the presence and severity of 15 items including facial flushing, sleep disturbance, and feeling angry or bad tempered in those with MS with a confirmed relapse. However, patients did not clearly participate in the development of the questionnaire and the psychometric properties of the questionnaire were not evaluated. The SLE Steroid Questionnaire measured 50 items across 7 domains. Although patients were involved throughout the development process, this PRO has not been tested across a large population of patients, and psychometric testing and adequate measurement properties have not been demonstrated.

Cross-sectional survey of GC AE from the patient perspective on 2 continents. To complement the survey performed and previously presented within an Australian tertiary rheumatology clinic, the same survey was subsequently administered to both GC users and nonusers from the rheumatoid arthritis (RA) database at the Hospital for Special Surgery (HSS; New York) ${ }^{9}$. The questionnaire included a checklist of 19 known AE and asked participants to rate the 3 "worst" AE. Similar results were found in the HSS GC users when compared with the initial Australian cohort, suggesting that the patient's perception of GC AE appear similar despite cultural and geographic differences. The most frequent $\mathrm{AE}$ across the 2 groups of GC users were sleep disturbance, thin skin/easy bruising, and weight gain. Weight gain was described most frequently as the worst $\mathrm{AE}$ in both cohorts. Compared to the HSS GC nonusers, many GC AE were significantly more frequent among GC users, suggesting that these AE were due to the use of GC, rather than to other medications or the underlying disease itself

Qualitative assessment of GC use in RA. Patients with RA at the HSS with experience of GC use were invited to participate in a qualitative study to supplement the qualitative work that had already been conducted and reported on in antineutrophil cytoplasmic antibody-associated vasculitis (AAV), giant cell arteritis, and polymyalgia rheumatica, conditions in which both the dose as well as duration of GC may be significantly different from those in $\mathrm{RA}^{4,10}$. Eleven participants with RA ( 9 female) attended 1-to-1 semistructured interviews to describe the experience (benefits and harm) of taking GC. Ages ranged from 26-83 years. Eight participants were currently taking GC (range 2-20 mg daily of prednisone equivalent). Four themes emerged (Table 1). Overall, GC had been beneficial in the control of RA symptoms such as swelling and pain. However, this had "come at a price," the participants said, referring to the unintended physical and emotional effects of GC, such as weight gain and feelings of anger. Additionally, there was an acknowledgment of the necessity of GC use in certain contexts because of the need to be able to function for family and work purposes. Finally, there was uncertainty over attribution of potential symptoms solely to GC or to other disease-modifying antirheumatic drugs or to RA itself. Compared to the themes that emerged from the interviews conducted in AAV, similarities included the beneficial effects (which were quick), the adverse effects both physical and emotional, as well as the need to balance both in relation to the participants' current life situation. However, those with AAV voiced uncertainty regarding the dose-reduction process, whereas those with RA were at times uncertain whether particular effects were due to GC use.

SLR of the effect of GC from the patient perspective. To establish whether GC therapy carried similar effects in nonrheumatological inflammatory conditions, a further SLR was undertaken to identify the effects of systemic $\mathrm{GC}$ in adults across any condition in which systemic GC were used ${ }^{11}$. An academic librarian searched OVID EMBASE, OVID MEDLINE, PsycINFO, and CINAHL for articles published from inception to October 2017, related to 3 concepts: GC, the patient perspective, and AE. Inclusion criteria included systemic GC use for any indication in an adult population and both qualitative and quantitative research methodology. The initial search retrieved 1356 articles, of which 24 (18 quantitative, 6 qualitative) were deemed suitable for quality assessment and data extraction. Studies included the assessment of GC use across a variety of diseases both rheumatological (e.g., RA, vasculitis) and nonrheumatological (including asthma, inflammatory bowel disease, and MS). Four major themes emerged among the 71 discrete outcomes (Table 2): physical symptoms (44), psychological symptoms (18), effect on participation (6), and contextual factors (3). The metasynthesis of the qualitative work was richest for outcomes that had not been as well represented previously, including the effect on work/relationships, the cognitive load of debating the benefits and harm of GC use but also the sense of self-management and mastery of one's own disease, and the appropriate use of GC. Using a qualitative metasummary, frequency and intensity effect sizes will be calculated to identify those outcomes most prominently featured across all reviewed articles.

\section{DISCUSSION}

The session was well attended and insightful discussion took

Table 1. Description of themes from thematic analysis of interview transcripts.

Theme Key Points with Quotes

Benefits

Pain and swelling

- "...it does reduce the swelling. And when the swelling is reduced, the joints feel much better."

Return to functional activities

- 'If I can't move my hands, I can't take a shower, I can't wash my hair, I can't brush my teeth. So how do I get to work? For me that was debilitating...the prednisone allowed me to do that because I would feel the effects within 2-3 days..."

Challenges Physical (e.g., weight gain and recurrent infections)

- "I looked like a white whale with a harpoon in my hip... I looked like Moby Dick." "I had a respiratory infection a lot; I seem subject to those. I would get skin infections, I had to be so careful not to break my skin and things."

Emotional (e.g., anger and low mood)

- “...inside I felt like a terrorist. I really did. I could have killed somebody. I'm not kidding, I had a terrible temper..." "My parents ...for them to see me unhappy and just not feeling like myself, in that way, is really hard on them and it's hard on me..."

Necessity Frustration with need

- "I was angry that I had to take them. I did not want to take them, but I had no choice, because they do reduce the inflammation."

Attribution Unsure whether GC solely responsible

- “...when I'm taking all this...it's hard to precisely point to what's working and what's not working, what's good, what's bad..."

GC: glucocorticoid. 
Table 2. Outcomes with the most frequently reported outcome per theme.

\begin{tabular}{ll}
\hline Theme & Outcome \\
\hline Physical symptoms & Weight gain \\
& Sleep problems \\
& Skin changes \\
& Upper GI problems \\
& Cardiopulmonary \\
& Irritability and mood swings \\
& Depression \\
Psychological symptoms & Anxiety \\
& Hyperactivity and euphoria \\
& Process of debating GC use \\
& Effect on sexual relationships \\
& Effect on work \\
Participation & Effect on family \\
& Lack of support from community or media \\
Contextual factors & Self-management and mastery \\
& Lack of support from family and friends \\
\hline
\end{tabular}

GI: gastrointestinal; GC: glucocorticoid.

place among attendees and WG members, who identified issues to consider for future work, including:

1. The ongoing challenge of being able to clearly attribute an outcome to GC, rather than to the underlying disease or other medication(s).

2. How to create a PRO to be used across a broad range of rheumatic diseases in which GC use may be very different regarding dose, duration, and frequency.

3. How to integrate with the work of the Drug Safety WG and whether a generic core set for drug safety in addition to a GC-specific core set would be appropriate.

4. The importance of life context in determining the relative importance of a $\mathrm{GC}$ effect and how this can change over time.

5. That 3 main areas should be considered when assessing the effect of GC: the intended effects, the unintended effects, and the life context in which those effects take place.

A summary of both the presentation and subsequent discussion was also identified in cartoon form (Figure 1). Despite these issues, there was overall agreement that a PRO primarily concentrating on measuring the life effect of GC use is needed, and the following research agenda was developed:

1. Complete a Delphi exercise to prioritize outcomes.

2. Assess whether a different approach to the Delphi is needed to prioritize true GC effects (as opposed to the effects of the underlying disease or other medications).

3. Investigate novel ways of incorporating GC outcomes into the OMERACT onion ${ }^{5,6}$, because having the effect of a medication as the outcome may require some adaptation.

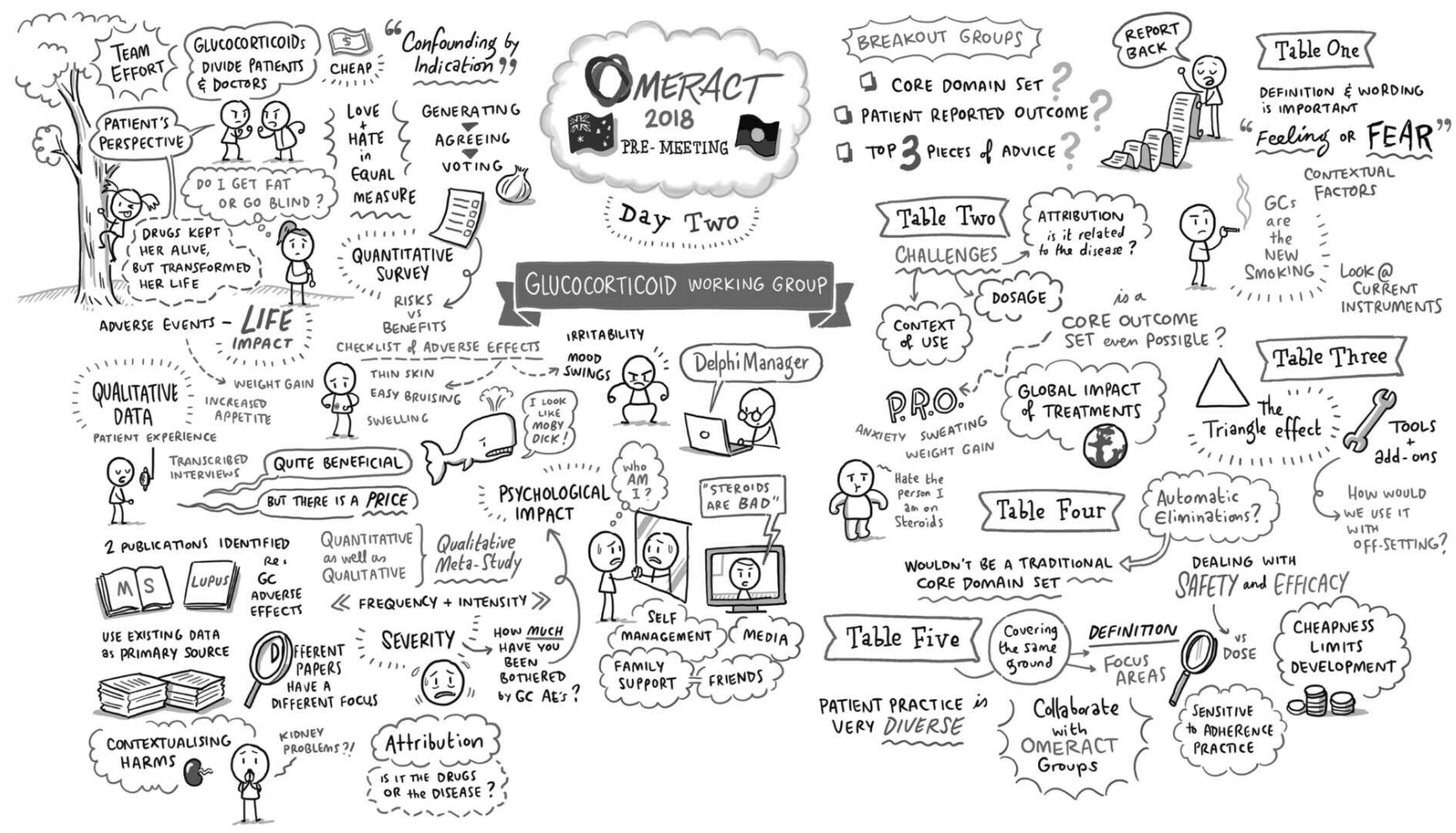

Figure 1. Summary of the Glucocorticoid Impact Special Interest Group session. OMERACT: Outcome Measures in Rheumatology; GC: glucocorticoid; AE: adverse effects; MS: multiple sclerosis. OMERACT logo from OMERACT; used with permission.

Personal non-commercial use only. The Journal of Rheumatology Copyright @ 2019 . All rights reserved. 
4. Develop a preliminary core domain set to be voted on at OMERACT 2020.

The ability to measure the effect of GC, both positive and negative, is crucial at a time when there are an increasing number of steroid-sparing agents requiring rigorous evaluation in clinical trials. Therefore, there is an unmet need to measure the outcomes of GC use from the patient perspective. Developing a core domain set to create such a PRO remains the goal of the WG.

\section{REFERENCES}

1. Laugesen K, Jørgensen JOL, Sørensen HT, Petersen I. Systemic glucocorticoid use in Denmark: A population-based prevalence study. BMJ Open 2017;7:e015237.

2. van Staa TP, Leufkens HG, Abenhaim L, Begaud B, Zhang B, Cooper C. Use of oral corticosteroids in the United Kingdom. QJM 2000;93:105-11.

3. Hoes JN, Jacobs JW, Verstappen SM, Bijlsma JW, Van der Heijden GJ. Adverse events of low- to medium-dose oral glucocorticoids in inflammatory diseases: a meta-analysis. Ann Rheum Dis 2009;68:1833-8.

4. Black RJ, Robson JC, Goodman SM, Hoon E, Lai LYH, Simon LS, et al. A patient-reported outcome measure for effect of glucocorticoid therapy in adults with inflammatory diseases is needed: Report from the OMERACT 2016 special interest group. J Rheumatol 2017;44:1754-8.
5. Boers M, Kirwan JR, Tugwell P, Beaton D, Bingham CO III, Conaghan PG, et al. The OMERACT Handbook. [Internet. Accessed May 17, 2017.] Available from: https://omeract.org/resources

6. Boers M, Kirwan JR, Wells G, Beaton D, Gossec L, d'Agostino MA, et al. Developing core outcome measurement sets for clinical trials: OMERACT filter 2.0. J Clin Epidemiol 2014;67:745-53.

7. Jongen PJ, Stavrakaki I, Voet B, Hoogervorst E, van Munster E, Linssen WH, et al. Patient-reported adverse effects of high-dose intravenous methylprednisolone treatment: a prospective web-based multi-center study in multiple sclerosis patients with a relapse. J Neurol 2016;263:1641-51

8. Mathias SD, Berry P, De Vries J, Askanase A, Pascoe K, Colwell $\mathrm{HH}$, et al. Development of the systemic lupus erythematosus steroid questionnaire (SSQ): a novel patient-reported outcome tool to assess the impact of oral steroid treatment. Health Qual Life Outcomes 2017;15:43.

9. Black RJ, Goodman SM, Ruediger C, Lester S, Mackie SL, Hill CL A survey of glucocorticoid adverse effects and benefits in rheumatic diseases: the patient perspective. J Clin Rheumatol 2017;23:416-20.

10. Robson JC, Dawson J, Cronholm PF, Ashdown S, Easley E, Kellom $\mathrm{KS}$, et al. Patient perceptions of glucocorticoids in anti-neutrophil cytoplasmic antibody-associated vasculitis. Rheumatol Int 2018;38:675-82.

11. Hill CL, Cheah JT, Goodman SM, Black RJ, Mackie SL. Patient's perspective of glucocorticoid use: A systematic review of quantitative and qualitative studies. PROSPERO 2018 CRD42018081620. [Internet. Accessed November 28, 2018.] Available from: www.crd.york.ac.uk/prospero/display_record.php?RecordID $=81620$ 